\section{P36 EFFECTIVENESS OF INTERVENTIONS TO IMPROVE SELF-MANAGEMENT FOR ADOLESCENTS AND YOUNG ADULTS WITH ALLERGIC CONDITIONS: A SYSTEMATIC REVIEW}

L Angier ${ }^{*},{ }^{2} \mathrm{C}$ Alviani, ${ }^{3} \mathrm{~T}$ Garriga, ${ }^{4} \mathrm{C}$ Moertz, ${ }^{5} \mathrm{M}$ Vazquez-Ortiz, ${ }^{2} \mathrm{G}$ Roberts. ${ }^{1}$ Psychology, Aston University, Birmingham, UK; ${ }^{2}$ Faculty of Medicine, University of Southampton, Southampton, UK; ${ }^{3}$ Facultativa Especialista Adjunta, Hospital Universitari Vall d'Hebron, Barcelona, Spain; ${ }^{4}$ Allergy Centre, Odense Universitetshospital, Odense, Denmark; ${ }^{5}$ Allergy Clinic, Imperial College NHS Healthcare Trust, London, UK

\subsection{6/bmjpo-2019-RCPCH-SAHM.40}

Aims This systematic review aimed to review the literature on interventions for improving self-management and wellbeing in adolescents and young adults (11-25 years) with allergic conditions, including asthma.

Methods A search strategy was constructed using Cochrane Database of Systematic Reviews, MEDLINE, Embase and Psychinfo. Studies had to be controlled trials or randomised control trials. Databases were searched to February 10, 2019. Quality assessments and data extraction were undertaken independently by two reviewers.

Results A total of 30 papers reporting data from 27 studies were included. Studies were conducted in the USA $(k=17)$; Netherlands $(k=2)$; Iran $(k=2)$; Australia $(k=2)$; Jordan $(\mathrm{k}=1)$; Canada $(\mathrm{k}=1)$; UK $(\mathrm{k}=1)$; Germany $(\mathrm{k}=1)$. Interventions were of 4 main types: psychological $(k=9)$; E-health $(\mathrm{k}=8)$; educational $(\mathrm{k}=4)$; peer led $(\mathrm{k}=5)$; with 1 intervention focused on breathing re-training. All interventions were for asthma. Psychological interventions used cognitive behavioural or motivational interviewing methods, emotional disclosure, stress management or problem solving to improve health outcomes. Significant improvements in the intervention group compared to the control group were found for self-esteem, quality of life, self-efficacy, coping strategies and mood alongside improvements in asthma symptoms. E-Health interventions included the use of webbased computer tailored information, telecommunication compressed videos or mobile applications. Significant improvements were seen across most studies in inhaler technique, adherence, quality of life and in asking questions about asthma medication, triggers and environmental control. Educational interventions included group sessions focusing on asthma prevention and management, individual coaching sessions and nurse-led asthma clinics. They demonstrated significantly improved management of asthma symptoms, improved controller medication use and increased use of a written management plan, reduction in symptoms and improved quality of life. The peer led interventions included the Triple A (Adolescent Asthma Action) programme and a peer-led camp based on the Power Breathing Programme. Improvements were found for self-efficacy, school absenteeism and quality of life.

Conclusion Although significant improvements were seen across all intervention types, many studies were small feasibility or pilot studies and no studies for allergic conditions other than asthma met the inclusion criteria. Large longitudinal interventional studies across the range of allergic conditions are required to strengthen the evidence base.

\section{P37 OPTIMISING ORAL ISOTRETINOIN DOSING FOR YOUNG PEOPLE WITH SEVERE ACNE}

S Elsarafy*, J Dobson, D Wood, J Ravenscroft. Paediatric Dermatology, Nottingham University Hospital, Nottingham, UK

\subsection{6/bmjpo-2019-RCPCH-SAHM.41}

Acne affects many young people and can have a profound effect on self-image. Treatment is based on a stepwise approach starting with topical treatments and escalating to oral treatments. Isotretinoin is a vitamin A derivative which is a very effective treatment for acne which is nodulo-cystic or scarring. Isotretinoin has a number of side effects, including reports of low mood and suicide. In the UK the pharmaceutical licence restricts prescribing to dermatologists. Published guidelines recommend a variety of different dosing schedules of oral isotretinoin for acne. Whilst there is no clear consensus on the optimal dosing strategy to reduce relapse, the available literature suggests $0.5-1.0 \mathrm{mg} / \mathrm{kg} / \mathrm{day}$ with a cumulative dose of $120-150 \mathrm{mg} / \mathrm{kg}$ and treatment duration of at least 6 months. In practice, dosing of isotretinoin may be influenced by tolerability of physical and psychological adverse effects. We present a quality improvement project using retrospective cases to evaluate our departmental isotretinoin dosing practice in young people to determine whether (1) we optimise dosing to reduce relapse based on published guidelines, (2) how screening and monitoring for physical and psychological health, in conjunction with acne related quality of life (QoL), influenced clinicians 'dosing decisions. Forty-two young people were included. Results showed 53\% patients reached a cumulative dose of $120 \mathrm{mg} / \mathrm{kg}$, and treatment duration was less than 6 months in 36\%. Reasons for dose limitation were physical (26\%) and psychological (21\%) adverse effects. Measures of depressive symptoms improved in $74 \%$ and acne related QoL improved in $89.5 \%$ of cases at the end of treatment. Our data show that target total dose, dose duration and peak dose was not achieved in many of our patients and that physical or psychological adverse effects are limiting factors in achieving therapeutic targets. No serious adverse effects were reported and the measures of depressive symptoms and acne related quality of life improved during treatment. Uncertainty remains as to how to optimise dosing to reduce relapse rate. Further research is needed to determine the relapse rate of adolescence acne following isotretinoin treatment and the optimal dosing strategy to reduce relapse including the possibility of low dose isotretinoin regimens.

\section{P38 A TRANSITION EVENING IS AN EFFECTIVE WAY TO DELIVER THE NICE QUALITY STANDARD AND IS POPULAR WITH SERVICE USERS}

AH Scott*, E Sheppard, A Critchlow, N Butler. Paediatric Respiratory Medicine, Sheffield Children's Hospital, Sheffield, UK

\subsection{6/bmjpo-2019-RCPCH-SAHM.42}

Aims With a population of almost 500 general respiratory patients aged between 14 and 18 years, we developed a transition evening to deliver patient-centred, quality care. Our aim 